\title{
Topological Soliton Solution and Bifurcation Analysis of the Klein-Gordon-Zakharov Equation in $(1+1)$-Dimensions with Power Law Nonlinearity
}

\author{
Ming Song, ${ }^{1}$ Bouthina S. Ahmed, ${ }^{2}$ and Anjan Biswas ${ }^{3,4}$ \\ ${ }^{1}$ Department of Mathematics, Yuxi Normal University, Yuxi, Yunnan 653100, China \\ 2 Department of Mathematics, Girls' College, Ain Shams University, Cairo 11757, Egypt \\ ${ }^{3}$ Department of Mathematical Sciences, Delaware State University, Dover, DE 19901-2277, USA \\ ${ }^{4}$ Department of Mathematics, Faculty of Science, King Abdulaziz University, Jeddah, Saudi Arabia \\ Correspondence should be addressed to Anjan Biswas; biswas.anjan@gmail.com
}

Received 22 November 2012; Accepted 11 December 2012

Academic Editor: Abdul Hamid Kara

Copyright (C) 2013 Ming Song et al. This is an open access article distributed under the Creative Commons Attribution License, which permits unrestricted use, distribution, and reproduction in any medium, provided the original work is properly cited.

This paper addresses the Klein-Gordon-Zakharov equation with power law nonlinearity in $(1+1)$-dimensions. The integrability aspect as well as the bifurcation analysis is studied in this paper. The numerical simulations are also given where the finite difference approach was utilized. There are a few constraint conditions that naturally evolve during the course of derivation of the soliton solutions. These constraint conditions must remain valid in order for the soliton solution to exist. For the bifurcation analysis, the phase portraits are also given.

\section{Introduction}

The theory of nonlinear evolution equations (NLEEs) has come a long way in the past few decades [1-20]. Many of the NLEEs are pretty well known in the area of theoretical physics and applied mathematics. A few of them are the nonlinear Schrödinger's equation, Korteweg-de Vries (KdV) equation, sine-Gordon equation which appear in nonlinear optics, fluid dynamics, and theoretical physics, respectively. It is also very common to come across several combo NLEEs such as the Schrödinger-KdV equation, Klein-Gordon-Zakharov (KGZ) equation, and many others that are also studied in the context of applied mathematics and theoretical physics. This paper is going to focus on the KGZ equation that will be studied with power law nonlinearity in $(1+1)$-dimensions.

The integrability aspects and the bifurcation analysis will be the main focus of this paper. The ansatz method will be applied to obtain the topological 1-soliton solution, also known as the shock wave solution, to this equation. The constraint conditions will be naturally formulated in order for the soliton solution to exist. Subsequently, the bifurcation analysis will be carried out for this paper. In this context, the phase portraits will be given. Additionally, other traveling wave solutions will be enumerated. Finally, the numerical simulation to the equation will be given. The finite difference scheme will also be given.

\section{Mathematical Analysis}

The KGZ equation with power law nonlinearity in $(1+1)$ dimensions that are going to be studied in this paper is given by [6]

$$
\begin{gathered}
q_{t t}-k^{2} q_{x x}+a q+b r q+c|q|^{2 n} q=0, \\
r_{t t}-k^{2} r_{x x}=d\left(|q|^{2 n}\right)_{x x},
\end{gathered}
$$

where $a, b, c, d$, and $k$ are real valued constants. Additionally $q(x, t)$ is a complex valued dependent variable and $r(x, t)$ is a real valued dependent variable. This section will focus on extracting the shock wave solutions to the KGZ equation (1) and (2) that are also known as topological soliton solution. 
Therefore the starting hypothesis will be

$$
\begin{gathered}
q(x, t)=A_{1} \tanh ^{p_{1}} \tau e^{i \phi}, \\
r(x, t)=A_{2} \tanh ^{p_{2}} \tau,
\end{gathered}
$$

where

$$
\tau=B(x-v t)
$$

Here, in (3) and (4) $A_{1}, A_{2}$ and $B$ are free parameters, while $v$ is the velocity of the soliton. The unknown exponents $p_{1}$ and $p_{2}$ will be determined, in terms of $n$ by the aid of balancing principle. The phase component of (3) is given by

$$
\phi=-\kappa x+\omega t+\theta
$$

where $\kappa$ represents the soliton frequency, $\omega$ is the soliton wave number, and $\theta$ is the phase constant. Substituting the hypothesis (3) and (4) into (1) and (2) yields

$$
\begin{aligned}
& p_{1}\left(p_{1}-1\right)\left(v^{2}-k^{2}\right) B^{2} \tanh ^{p_{1}-2} \tau \\
& -2 i p_{1}\left(v \omega-\kappa^{2} k^{2}\right) B \tanh ^{p_{1}-1} \tau \\
& -\left\{2 p_{1}^{2}\left(v^{2}-k^{2}\right) B^{2}+\omega^{2}+K^{2} \kappa^{2}\right\} \tanh ^{p_{1}} \tau \\
& +2 i p_{1}\left(v \omega-\kappa^{2} k^{2}\right) B \tanh ^{p_{1}+1} \tau \\
& +p_{1}\left(p_{1}+1\right)\left(v^{2}-k^{2}\right) B^{2} \tanh ^{p_{1}+2} \tau+a \tanh ^{p_{1}} \tau \\
& +b A_{2} \tanh ^{p_{1}+p_{2}} \tau+c A_{1}^{2 n} \tanh ^{(2 n+1) p_{1}} \tau=0, \\
& p_{2}\left(p_{2}-1\right)\left(v^{2}-k^{2}\right) A_{2} B^{2} \tanh ^{p_{2}-2} \tau \\
& -2 p_{2}^{2}\left(v^{2}-K^{2}\right) A_{2} B^{2} \tanh ^{p_{2}} \tau \\
& +p_{2}\left(p_{2}+1\right)\left(v^{2}-k^{2}\right) A_{2} B^{2} \tanh ^{p_{2}+2} \tau \\
& -d A_{1}^{2 n} B^{2}\left\{2 n p_{1}\left(2 n p_{1}-1\right) \tanh ^{2 n p_{1}-2} \tau-8 n^{2} p_{1}^{2} \tanh ^{2 n p_{1}} \tau\right. \\
& \left.+2 n p_{1}\left(2 n p_{1}+1\right) \tanh ^{2 n p_{1}+2} \tau\right\}=0,
\end{aligned}
$$

respectively. Now, splitting (7) into two real and imaginary parts gives

$$
\begin{aligned}
& p_{1}\left(p_{1}-1\right)\left(v^{2}-k^{2}\right) B^{2} \tanh ^{p_{1}-2} \tau \\
&-\left\{2 p_{1}^{2}\left(v^{2}-k^{2}\right) B^{2}+\omega^{2}+k^{2} \kappa^{2}\right\} \tanh ^{p_{1}} \tau \\
&+ p_{1}\left(p_{1}+1\right)\left(v^{2}-k^{2}\right) B^{2} \tanh ^{p_{1}+2} \tau+a \tanh ^{p_{1}} \tau \\
&+ b A_{2} \tanh ^{p_{1}+p_{2}} \tau+c A_{1}^{2 n} \tanh ^{(2 n+1) p_{1}} \tau=0, \\
& 2 i p_{1}\left(v \omega-\kappa^{2} k^{2}\right) B \tanh ^{p_{1}-1} \tau \\
&-2 i p_{1}\left(v \omega-\kappa^{2} k^{2}\right) B \tanh ^{p_{1}+1} \tau=0 .
\end{aligned}
$$

From (9), equating the exponents $p_{1}+p_{2}$ and $p_{1}+2$ gives

$$
p_{2}=2 \text {, }
$$

and then equating $(2 n+1) p_{1}$ with $p_{1}+2$ gives

$$
p_{1}=\frac{1}{n} \text {. }
$$

Finally, equating the exponent pairs $(2 n+1) p_{1}$ and $p_{1}+p_{2}$ gives

$$
p_{2}=2 n p_{1}
$$

Now the values of $p_{1}$ and $p_{2}$ from (11) and (12) satisfy (13).

Finally, equating the coefficients of the linearly independent functions $\tanh ^{p_{1} \pm j} \tau, j= \pm 1,0, \pm 2$ in (9) and (10) to zero gives

$$
\begin{gathered}
\left(v \omega-\kappa^{2} k^{2}\right) B=0, \\
2\left(v^{2}-k^{2}\right) B^{2}+n^{2}\left(\omega^{2}+\kappa^{2} k^{2}-a\right)=0, \\
(n+1)\left(v^{2}-k^{2}\right) B^{2}+n^{2}\left(b A_{2}+c A_{1}^{2 n}\right)=0 .
\end{gathered}
$$

Again, equating the coefficients of the linearly independent functions $\tanh ^{p_{2} \pm j} \tau, j= \pm 1, \pm 2$ in (8) to zero implies

$$
\left(v^{2}-k^{2}\right) A_{2}-d A_{1}^{2 n}=0
$$

Solving (14)-(15) we get

$$
\begin{gathered}
\omega=\frac{k^{2} \kappa^{2}}{v}, \\
B=n \sqrt{\frac{a-k^{2} \kappa^{2}-\omega^{2}}{2\left(v^{2}-k^{2}\right)}}, \\
A_{1}=\left[\frac{(n+1)\left(v^{2}-k^{2}\right)\left(\omega^{2}-a-\kappa^{2} k^{2}\right) d}{2\left\{c\left(v^{2}-k^{2}\right)+b d\right\}}\right]^{1 / 2 n}, \\
A_{2}=\frac{d(n+1)\left(\omega^{2}-a-k^{2} \kappa^{2}\right)}{2\left\{c\left(v^{2}-k^{2}\right)+b d\right\}} .
\end{gathered}
$$

The relations (17), (18), and (19) introduce the restrictions given by

$$
\begin{gathered}
\left(a-k^{2} \kappa^{2}-\omega^{2}\right)\left(v^{2}-k^{2}\right)>0 \\
d\left(v^{2}-k^{2}\right)\left(\omega^{2}-a-K^{2} \kappa^{2}\right)\left\{c\left(v^{2}-k^{2}\right)+b d\right\}>0 \\
c\left(v^{2}-k^{2}\right)+b d \neq 0 .
\end{gathered}
$$

Thus the topological solution of the $q$ and $r$ wave functions are given:

$$
\begin{gathered}
q(x, t)=A_{1} \tanh ^{1 / n}[B(x-v t)] e^{i(-\kappa x+\omega t+\theta)}, \\
r(x, t)=A_{2} \tanh ^{2}[B(x-v t)] .
\end{gathered}
$$




\section{Bifurcation Analysis}

This section will carry out the bifurcation analysis of the Klein-Gordon-Zakharov equation with power law nonlinearity. Initially, the phase portraits will be obtained and the corresponding qualitative analysis will be discussed. Several interesting properties of the solution structure will be obtained based on the parameter regimes. Subsequently, the traveling wave solutions will be discussed from the bifurcation analysis.

3.1. Phase Portraits and Qualitative Analysis. We assume that the traveling wave solutions of (1) and (2) are of the form

$$
\begin{array}{rlrl}
q(x, t) & =e^{i \eta} \varphi(\xi), & & r(x, t)=\psi(\xi), \\
\eta & =m x+l t, & \xi=p x-v t,
\end{array}
$$

where $\varphi(\xi)$ and $\psi(\xi)$ are real functions, $m, l, p$, and $v$ are real constants.

Substituting (22) and (23) into (1) and (2), we find that $p=-v l / m k^{2}, \varphi$ and $\phi$ satisfy the following system:

$$
\begin{gathered}
\left(v^{2}-k^{2} p^{2}\right) \varphi^{\prime \prime}-\left(l^{2}-k^{2} m^{2}-a\right) \varphi+b \varphi \psi+c \varphi^{2 n+1}=0 \\
\left(v^{2}+k^{2} p^{2}\right) \psi^{\prime \prime}-d p^{2}\left(\varphi^{2 n}\right)^{\prime \prime}=0 .
\end{gathered}
$$

Integrating (25) twice and letting the first integral constant be zero, we have

$$
\psi=\frac{d p^{2} \varphi^{2 n}}{v^{2}-k^{2} p^{2}}+g, \quad v^{2}-k^{2} p^{2} \neq 0,
$$

where $g$ is the second integral constant.

Substituting (26) into (24), we have

$$
\begin{gathered}
\left(v^{2}-k^{2} p^{2}\right) \varphi^{\prime \prime}-\left(l^{2}-k^{2} m^{2}-a-b g\right) \varphi \\
+\left(c+\frac{b d p^{2}}{v^{2}-k^{2} p^{2}}\right) \varphi^{2 n+1}=0 .
\end{gathered}
$$

To facilitate discussions, we let

$$
\begin{gathered}
\delta=\frac{b d p^{2}+c\left(v^{2}-k^{2} p^{2}\right)}{\left(v^{2}-k^{2} p^{2}\right)^{2}}, \\
\theta=\frac{l^{2}-k^{2} m^{2}-a-b g}{v^{2}-k^{2} p^{2}} .
\end{gathered}
$$

Letting $\varphi^{\prime}=z$, then we get the following planar system:

$$
\begin{gathered}
\frac{\mathrm{d} \varphi}{\mathrm{d} \xi}=z, \\
\frac{\mathrm{d} z}{\mathrm{~d} \xi}=-\delta \varphi^{2 n+1}+\theta \varphi .
\end{gathered}
$$

Obviously, the above system (30) is a Hamiltonian system with Hamiltonian function

$$
H(\varphi, z)=z^{2}+\frac{\delta}{n+1} \varphi^{2 n+2}-\theta \varphi^{2} .
$$

In order to investigate the phase portrait of (30), set

$$
f(\varphi)=-\delta \varphi^{2 n+1}+\theta \varphi .
$$

Obviously, when $\delta \theta>0, f(\varphi)$ has three zero points, $\varphi_{-}, \varphi_{0}$, and $\varphi_{+}$, which are given as follows:

$$
\varphi_{-}=-\left(\frac{\theta}{\delta}\right)^{1 / 2 n}, \quad \varphi_{0}=0, \quad \varphi_{+}=\left(\frac{\theta}{\delta}\right)^{1 / 2 n} .
$$

When $\delta \theta \leqslant 0, f(\varphi)$ has only one zero point

$$
\varphi_{0}=0 .
$$

Letting $\left(\varphi_{i}, 0\right)$ be one of the singular points of system (30), then the characteristic values of the linearized system of system (30) at the singular points $\left(\varphi_{i}, 0\right)$ are

$$
\lambda_{ \pm}= \pm \sqrt{f^{\prime}\left(\varphi_{i}\right)} .
$$

From the qualitative theory of dynamical systems, we know the following.

(I) If $f^{\prime}\left(\varphi_{i}\right)>0,\left(\varphi_{i}, 0\right)$ is a saddle point.

(II) If $f^{\prime}\left(\varphi_{i}\right)<0,\left(\varphi_{i}, 0\right)$ is a center point.

(III) If $f^{\prime}\left(\varphi_{i}\right)=0,\left(\varphi_{i}, 0\right)$ is a degenerate saddle point.

Therefore, we obtain the bifurcation phase portraits of system (30) in Figure 1.

Let

$$
H(\varphi, z)=h,
$$

where $h$ is Hamiltonian.

Next, we consider the relations between the orbits of (30) and the Hamiltonian $h$.

Set

$$
h^{*}=\left|H\left(\varphi_{+}, 0\right)\right|=\left|H\left(\varphi_{-}, 0\right)\right| .
$$

According to Figure 1, we get the following propositions.

Proposition 1. Suppose that $\delta>0$ and $\theta>0$, one has the following.

(I) When $h \leqslant-h^{*}$, system (30) does not have any closed orbits.

(II) When $-h^{*}<h<0$, system (30) has two periodic orbits $\Gamma_{1}$ and $\Gamma_{2}$.

(III) When $h=0$, system (30) has two homoclinic orbits $\Gamma_{3}$ and $\Gamma_{4}$.

(IV) When $h>0$, system (30) has a periodic orbit $\Gamma_{5}$.

Proposition 2. Suppose that $\delta<0$ and $\theta<0$, one has the following.

(I) When $h<0$ or $h>h^{*}$, system (30) does not have any closed orbits.

(II) When $0<h<h^{*}$, system (30) has three periodic orbits $\Gamma_{6}, \Gamma_{7}$, and $\Gamma_{8}$. 


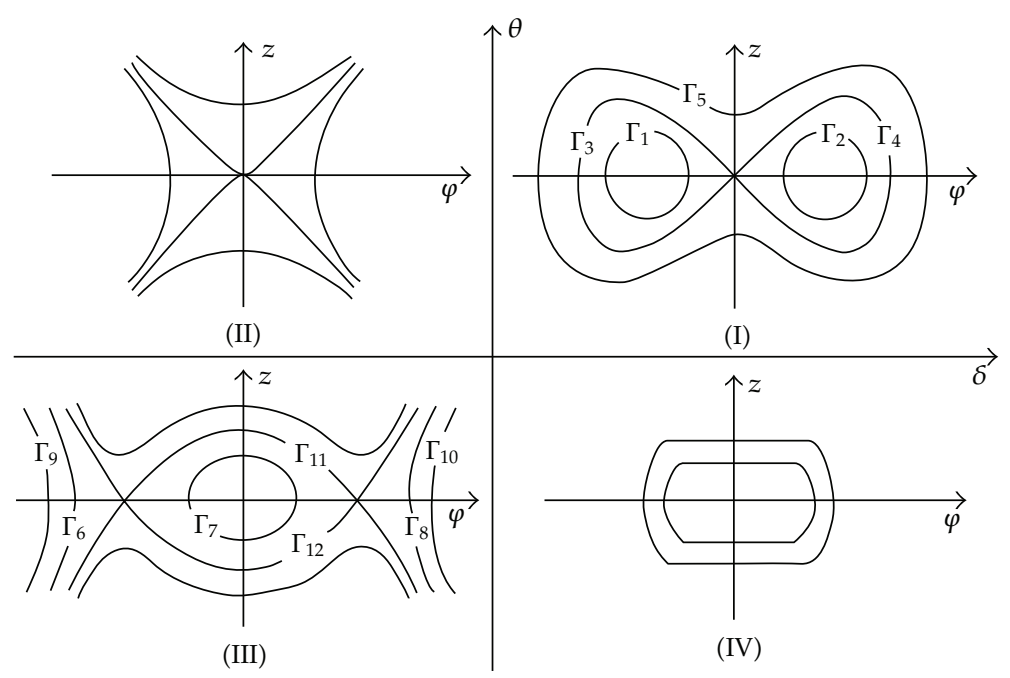

Figure 1: The bifurcation phase portraits of system (30). (I) $\delta>0, \theta>0$, (II) $\delta<0, \theta \geqslant 0$, (III) $\delta<0, \theta<0$, (IV) $\delta>0, \theta \leqslant 0$.

(III) When $h=0$, system (30) has two periodic orbits $\Gamma_{9}$ and $\Gamma_{10}$.

(IV) When $h=h^{*}$, system (30) has two heteroclinic orbits $\Gamma_{11}$ and $\Gamma_{12}$.

Proposition 3. (I) When $\delta>0, \theta \geqslant 0$ and $h>0$, system (30) has a periodic orbits.

(II) When $\delta<0, \theta \leqslant 0$, system (30) does have not any closed orbits.

From the qualitative theory of dynamical systems, we know that a smooth solitary wave solution of a partial differential system corresponds to a smooth homoclinic orbit of a traveling wave equation. A smooth kink wave solution or a unbounded wave solution corresponds to a smooth heteroclinic orbit of a traveling wave equation. Similarly, a periodic orbit of a traveling wave equation corresponds to a periodic traveling wave solution of a partial differential system. According to the above analysis, we have the following propositions.

Proposition 4. If $\delta>0$ and $\theta>0$, one has the following.

(I) When $-h^{*}<h<0$, (1) and (2) have two periodic wave solutions (corresponding to the periodic orbits $\Gamma_{1}$ and $\Gamma_{2}$ in Figure 1).

(II) When $h=0$, (1) and (2) have two solitary wave solutions (corresponding to the homoclinic orbits $\Gamma_{3}$ and $\Gamma_{4}$ in Figure 1).

(III) When $h>0$, (1) and (2) have two periodic wave solutions (corresponding to the periodic orbit $\Gamma_{5}$ in Figure 1).

Proposition 5. If $\delta<0$ and $\theta<0$, one has the following.

(I) When $0<h<h^{*}$, (1) and (2) have two periodic wave solutions (corresponding to the periodic orbit $\Gamma_{7}$ in Figure 1) and two periodic blow-up wave solutions (corresponding to the periodic orbits $\Gamma_{6}$ and $\Gamma_{8}$ in Figure 1).

(II) When $h=0$, (1) and (2) have periodic blow-up wave solutions (corresponding to the periodic orbits $\Gamma_{9}$ and $\Gamma_{10}$ in Figure 1).

(III) When $h=h^{*}$, (1) and (2) have two kink profile solitary wave solutions. (corresponding to the heteroclinic orbits $\Gamma_{11}$ and $\Gamma_{12}$ in Figure 1).

3.2. Exact Traveling Wave Solutions. Firstly, we will obtain the explicit expressions of traveling wave solutions for (1) and (2) when $\delta>0$ and $\theta>0$. From the phase portrait, we see that there are two symmetric homoclinic orbits $\Gamma_{3}$ and $\Gamma_{4}$ connected at the saddle point $(0,0)$. In $(\varphi, z)$-plane the expressions of the homoclinic orbits are given as

$$
z= \pm \sqrt{\frac{\delta}{n+1}} \varphi \sqrt{-\varphi^{2 n}+\frac{(n+1) \theta}{\delta}} .
$$

Substituting (38) into $\mathrm{d} \varphi / \mathrm{d} \xi=z$ and integrating them along the orbits $\Gamma_{3}$ and $\Gamma_{4}$, we have

$$
\begin{aligned}
& \pm \int_{\varphi_{1}}^{\varphi} \frac{1}{s \sqrt{-s^{2 n}+(n+1) \theta / \delta}} \mathrm{d} s=\sqrt{\frac{\delta}{n+1}} \int_{0}^{\xi} \mathrm{d} s, \\
& \pm \int_{\varphi_{2}}^{\varphi} \frac{1}{s \sqrt{-s^{2 n}+(n+1) \theta / \delta}} \mathrm{d} s=\sqrt{\frac{\delta}{n+1}} \int_{0}^{\xi} \mathrm{d} s,
\end{aligned}
$$

where $\varphi_{1}=-((n+1) \theta / \delta)^{1 / 2 n}$ and $\varphi_{2}=((n+1) \theta / \delta)^{1 / 2 n}$.

Completing the above integrals we obtain

$$
\begin{gathered}
\varphi=\left(\sqrt{\frac{(n+1) \theta}{\delta}} \operatorname{sech} n \sqrt{\theta} \xi\right)^{1 / n} \\
\varphi=-\left(\sqrt{\frac{(n+1) \theta}{\delta}} \operatorname{sech} n \sqrt{\theta} \xi\right)^{1 / n} .
\end{gathered}
$$


Noting (22), (23), and (26), we get the following solitary wave solutions:

$$
\begin{gathered}
q_{1}(x, y, t)=e^{i \eta}\left(\sqrt{\frac{(n+1) \theta}{\delta}} \operatorname{sech} n \sqrt{\theta} \xi\right)^{1 / n}, \\
r_{1}(x, y, t)=\frac{-(n+1) \beta \theta(\operatorname{sech} n \sqrt{\theta} \xi)^{2}}{\delta\left(p^{2}+m^{2}\right)}+g, \\
q_{2}(x, y, t)=-e^{i \eta}\left(\sqrt{\frac{(n+1) \theta}{\delta}} \operatorname{sech} n \sqrt{\theta} \xi\right)^{1 / n}, \\
r_{2}(x, y, t)=\frac{-(n+1) \beta \theta(\operatorname{sech} n \sqrt{\theta} \xi)^{2}}{\delta\left(p^{2}+m^{2}\right)}+g,
\end{gathered}
$$

where $\delta$ is given by (28), $\theta$ is given by (29), $\eta=m x+l t$, and $\xi=p x-v t$.

Secondly, we will obtain the explicit expressions of traveling wave solutions for (1) and (2) when $\delta<0$ and $\theta<0$. From the phase portrait, we note that there are two special orbits $\Gamma_{9}$ and $\Gamma_{10}$, which have the same Hamiltonian with that of the center point $(0,0)$. In $(\varphi, z)$-plane the expressions of the orbits are given as

$$
z= \pm \sqrt{-\frac{\delta}{n+1}} \varphi \sqrt{\varphi^{2 n}-\frac{(n+1) \theta}{\delta}}
$$

Substituting (42) into $\mathrm{d} \varphi / \mathrm{d} \xi=z$ and integrating them along the two orbits $\Gamma_{9}$ and $\Gamma_{10}$, it follows that

$$
\begin{aligned}
& \pm \int_{\varphi}^{+\infty} \frac{1}{s \sqrt{s^{2 n}-(n+1) \theta / \delta}} \mathrm{d} s=\sqrt{-\frac{\delta}{n+1}} \int_{0}^{\xi} \mathrm{d} s \\
& \pm \int_{\varphi_{4}}^{\varphi} \frac{1}{s \sqrt{s^{2 n}-(n+1) \theta / \delta}} \mathrm{d} s=\sqrt{-\frac{\delta}{n+1}} \int_{0}^{\xi} \mathrm{d} s,
\end{aligned}
$$

where $\varphi_{4}=((n+1) \theta / \delta)^{1 / 2 n}$.

Completing the above integrals we obtain

$$
\begin{aligned}
& \varphi= \pm\left(\sqrt{\frac{(n+1) \theta}{\delta}} \csc n \sqrt{-\theta} \xi\right)^{1 / n} \\
& \varphi= \pm\left(\sqrt{\frac{(n+1) \theta}{\delta}} \sec n \sqrt{-\theta} \xi\right)^{1 / n} .
\end{aligned}
$$

Noting (22), (23), and (26), we get the following periodic blow-up wave solutions:

$$
\begin{gathered}
q_{3}(x, y, t)= \pm e^{i \eta\left(\sqrt{\frac{(n+1) \theta}{\delta}} \csc n \sqrt{-\theta} \xi\right)^{1 / n}} \\
r_{3}(x, y, t)=\frac{-(n+1) \beta \theta(\csc n \sqrt{-\theta} \xi)^{2}}{\delta\left(p^{2}+m^{2}\right)}+g \\
q_{4}(x, y, t)= \pm e^{i \eta\left(\sqrt{\frac{(n+1) \theta}{\delta}} \sec n \sqrt{-\theta} \xi\right)^{1 / n}} \\
r_{4}(x, y, t)=\frac{-(n+1) \beta \theta(\sec n \sqrt{-\theta} \xi)^{2}}{\delta\left(p^{2}+m^{2}\right)}+g
\end{gathered}
$$

where $\delta$ is given by (28), $\theta$ is given by (29), $\eta=m x+l t$, and $\xi=p x-v t$.

\section{Numerical Simulation}

We decompose the function $q$ in (1) in the form

$$
q=u+i v
$$

Substituting in (1) and (2) we have

$$
\begin{gathered}
u_{t t}-k^{2} u_{x x}+a u+b r v+c\left(u^{2}+v^{2}\right)^{n} u=0, \\
v_{t t}-k^{2} v_{x x}+a v+b r v+c\left(u^{2}+v^{2}\right)^{n} v=0, \\
r_{t t}-k^{2} u_{x x}-\alpha\left(u^{2}+v^{2}\right)_{x x}^{n}=0 .
\end{gathered}
$$

We assume that $u_{m}^{n}, v_{m}^{n}, r_{m}^{n}$ is the exact solution and $U_{m}^{n}, V_{m}^{n}$, $R_{m}^{n}$ is the approximate solution at the grid point $\left(x_{m}, t_{n}\right)$. The proposed scheme can be displayed as

$$
\begin{gathered}
\frac{1}{k^{2}} \delta_{t}^{2} U_{m}^{n}-\frac{k^{2}}{h^{2}} \delta_{x}^{2} U_{m}^{n}+a U_{m}^{n}+b R_{m, n} U_{m}^{n} \\
+c\left(\left(U_{m}^{n}\right)^{2}+\left(U_{m}^{n}\right)^{2}\right)^{n} U_{m}^{n}=0 \\
\frac{1}{k^{2}} \delta_{t}^{2} V_{m}^{n}-\frac{k^{2}}{h^{2}} \delta_{x}^{2} V_{m}^{n}+a V_{m}^{n}+b R_{m, n} V_{m}^{n} \\
+c\left(\left(U_{m}^{n}\right)^{2}+\left(V_{m}^{n}\right)^{2}\right)^{n} V_{m}^{n}=0 \\
\frac{1}{k^{2}} \delta_{t}^{2} R_{m}^{n}-\frac{k^{2}}{h^{2}} \delta_{x}^{2} R_{m}^{n}+\alpha\left(\left(U_{m}^{n}\right)^{2}+\left(V_{m}^{n}\right)^{2}\right)_{x x}^{n}=0
\end{gathered}
$$

where

$$
\begin{aligned}
& \delta_{t}^{2} U_{m}^{n}=U_{m}^{n+1}-2 U_{m}^{n}+U_{m}^{n-1}, \\
& \delta_{x}^{2} U_{m}^{n}=U_{m+1}^{n}-2 U_{m}^{n}+U_{m-1}^{n} .
\end{aligned}
$$



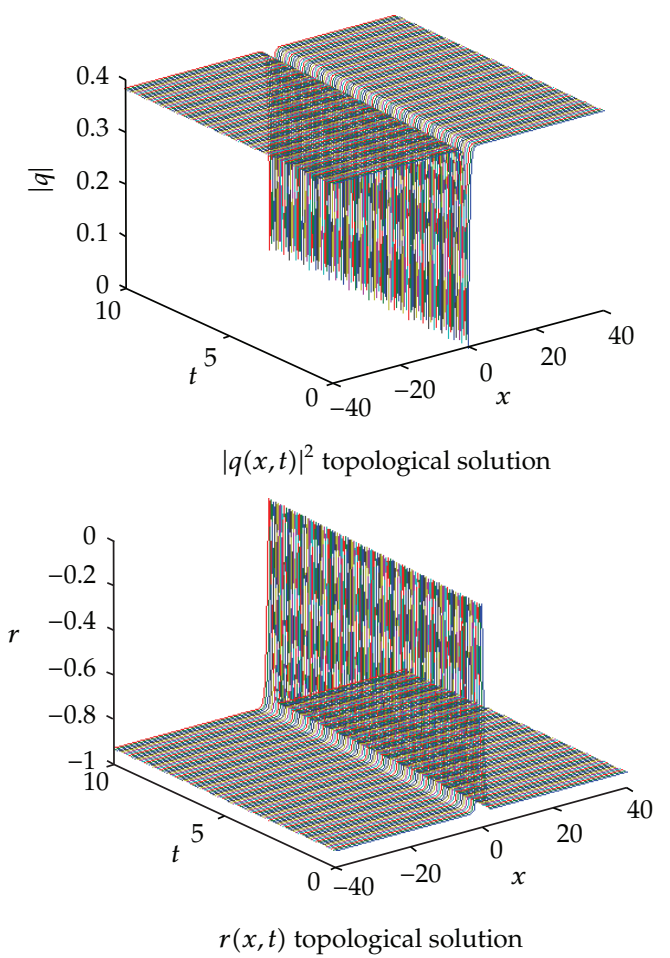

FIgURE 2: Topological solution for the Klein-Gordon-Zakharov equations.

The similar notation for $\delta_{t}^{2} V_{m}^{n}, \delta_{x}^{2} V_{m}^{n}$ and $\delta_{t}^{2} R_{m}^{n}, \delta_{x}^{2} R_{m}^{n}$ are

$$
\begin{aligned}
& \delta_{t}^{2} V_{m}^{n}=V_{m}^{n+1}-2 V_{m}^{n}+V_{m}^{n-1}, \\
& \delta_{x}^{2} V_{m}^{n}=V_{m+1}^{n}-2 V_{m}^{n}+V_{m-1}^{n}, \\
& \delta_{t}^{2} R_{m}^{n}=R_{m}^{n+1}-2 R_{m}^{n}+R_{m}^{n-1}, \\
& \delta_{x}^{2} R_{m}^{n}=R_{m+1}^{n}-2 R_{m}^{n}+R_{m-1}^{n},
\end{aligned}
$$

The proposed scheme is implicit and can be easily solved by the fixed point method. The scheme is second order in space and time directions.

To get the numerical solution the initial conditions are taken from the exact solution (21). Figure 2 displays the numerical solutions of $|q(x, t)|$ and $r(x, t)$ at $n=1$, respectively. We choose the parameter

$$
\begin{gathered}
n=1, \quad K=1, \quad \kappa=0.5, \quad a=0.5, \quad b=0.5, \\
c=-0.5, \quad d=0.5, \quad c=0.5, \quad v=0.6 .
\end{gathered}
$$

\section{Conclusions}

This paper studied the KGZ equation in $(1+1)$-D with power law nonlinearity from three different avenues. First, the topological 1-soliton solution to the equation was determined by the aid of ansatz method. The by-product of this solution is a couple of constraint conditions that must remain valid in order for the solitons to exist. Subsequently, the bifurcation analysis is carried out for this equation that leads to the phase portraits and several other solutions to the equation, using the traveling wave hypothesis. This leads to the solitary waves and periodic singular waves. Finally, the numerical simulation that was conducted using finite difference scheme leads to the simulations for the topological soliton solutions.

These results are pretty complete in analysis. They are going to be extended in the future. An obvious way to expand or generalize these results is going to extend to $(2+1)$-D. These results will be reported soon. Another avenue to look into this equation further is to consider the perturbation terms and then obtain exact solution, and additionally study the perturbed KGZ equation using other tools of integrability. These include mapping method, Lie symmetries, expfunction method, and the $G^{\prime} / G$-expansion method. These will lead to a further plethora of solutions. Such results will be reported in the future. That is just a foot in the door.

\section{References}

[1] G. Ebadi and A. Biswas, "Application of the G'/G-expansion method for nonlinear diffusion equations with nonlinear source," Journal of the Franklin Institute, vol. 347, no. 7, pp. 1391$1398,2010$.

[2] G. Ebadi and A. Biswas, "The $\mathrm{G}^{\prime} / \mathrm{G}$ method and 1-soliton solution of the Davey-Stewartson equation," Mathematical and Computer Modelling, vol. 53, no. 5-6, pp. 694-698, 2011.

[3] D. Feng and J. Li, "Dynamics and bifurcations of travelling wave solutions of $\mathrm{R}(m, n)$ equations," Proceedings of the Indian Academy of Sciences, vol. 117, no. 4, pp. 555-574, 2007.

[4] Z. Gan and J. Zhang, "Instability of standing waves for KleinGordon-Zakharov equations with different propagation speeds in three space dimensions," Journal of Mathematical Analysis and Applications, vol. 307, no. 1, pp. 219-231, 2005.

[5] Z. Gan, B. Guo, and J. Zhang, "Instability of standing wave, global existence and blowup for the Klein-Gordon-Zakharov system with different-degree nonlinearities," Journal of Differential Equations, vol. 246, no. 10, pp. 4097-4128, 2009.

[6] M. S. Ismail and A. Biswas, "1-Soliton solution of the KleinGordon-Zakharov equation with power law nonlinearity," Applied Mathematics and Computation, vol. 217, no. 8, pp. 41864196, 2010.

[7] E. V. Krishnan and A. Biswas, "Solutions to the ZakharovKuznetsov equation with higher order nonlinearity by mapping and ansatz methods," Physics of Wave Phenomena, vol. 18, no. 4, pp. 256-261, 2010.

[8] E. V. Krishnan and Y.-Z. Peng, "Exact solutions to the combined KdV-mKdV equation by the extended mapping method," Physica Scripta, vol. 73, no. 4, article 405, 2006.

[9] J. Li, "Exact explicit travelling wave solutions for $(n+1)$ dimensional Klein-Gordon-Zakharov equations," Chaos, Solitons and Fractals, vol. 34, no. 3, pp. 867-871, 2007.

[10] L. Chen, "Orbital stability of solitary waves for the KleinGordon-Zakharov equations," Acta Mathematicae Applicatae Sinica, vol. 15, no. 1, pp. 54-64, 1999.

[11] N. Masmoudi and K. Nakanishi, "From the Klein-GordonZakharov system to a singular nonlinear Schrödinger system," Annales de l'Institut Henri Poincare (C) Non Linear Analysis, vol. 27, no. 4, pp. 1073-1096, 2010. 
[12] Y. Shang, Y. Huang, and W. Yuan, "New exact traveling wave solutions for the Klein-Gordon-Zakharov equations," Computers and Mathematics with Applications, vol. 56, no. 5, pp. 14411450, 2008.

[13] T. Wang, J. Chen, and L. Zhang, "Conservative difference methods for the Klein-Gordon-Zakharov equations," Journal of Computational and Applied Mathematics, vol. 205, no. 1, pp. 430-452, 2007.

[14] A. M. Wazwaz, "The extended tanh method for new compact and noncompact solutions for the KP-BBM and the ZK-BBM equations," Chaos, Solitons and Fractals, vol. 38, no. 5, pp. 15051516, 2008.

[15] L. Zhang, "Convergence of a conservative difference scheme for a class of Klein-Gordon-Schrödinger equations in one space dimension," Applied Mathematics and Computation, vol. 163, no. 1, pp. 343-355, 2005.

[16] J. Li and Z. Liu, "Smooth and non-smooth traveling waves in a nonlinearly dispersive equation," Applied Mathematical Modelling, vol. 25, no. 1, pp. 41-56, 2000.

[17] M. Song, X. Hou, and J. Cao, "Solitary wave solutions and kink wave solutions for a generalized KDV-mKDV equation," Applied Mathematics and Computation, vol. 217, no. 12, pp. 5942-5948, 2011.

[18] M. Song and Z. Liu, "Periodic wave solutions and their limits for the generalized KP-BBM equation," Journal of Applied Mathematics, vol. 2012, Article ID 363879, 25 pages, 2012.

[19] M. Song, "Application of bifurcation method to the generalized Zakharov equations," Abstract and Applied Analysis, vol. 2012, Article ID 308326, 8 pages, 2012.

[20] M. Song, Z. Liu, E. Zerrad, and A. Biswas, "Singular soliton solution and bifurcation analysis of Klein-Gordon equation with power law nonlinearity," Frontiers of Mathematics in China, vol. 8, no. 1, pp. 191-201, 2013. 


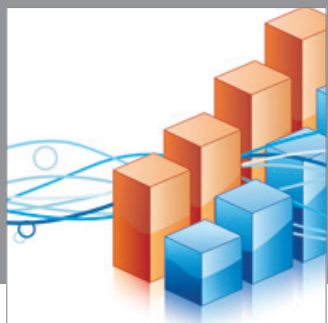

Advances in

Operations Research

mansans



The Scientific World Journal
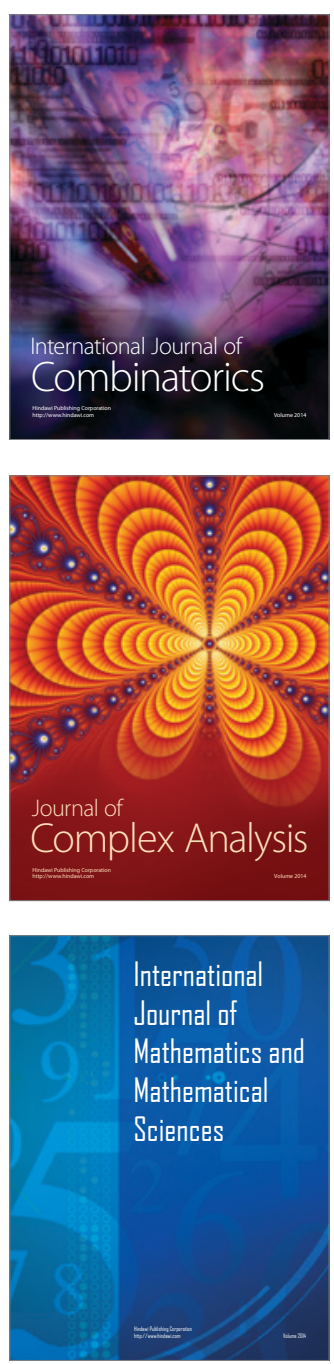
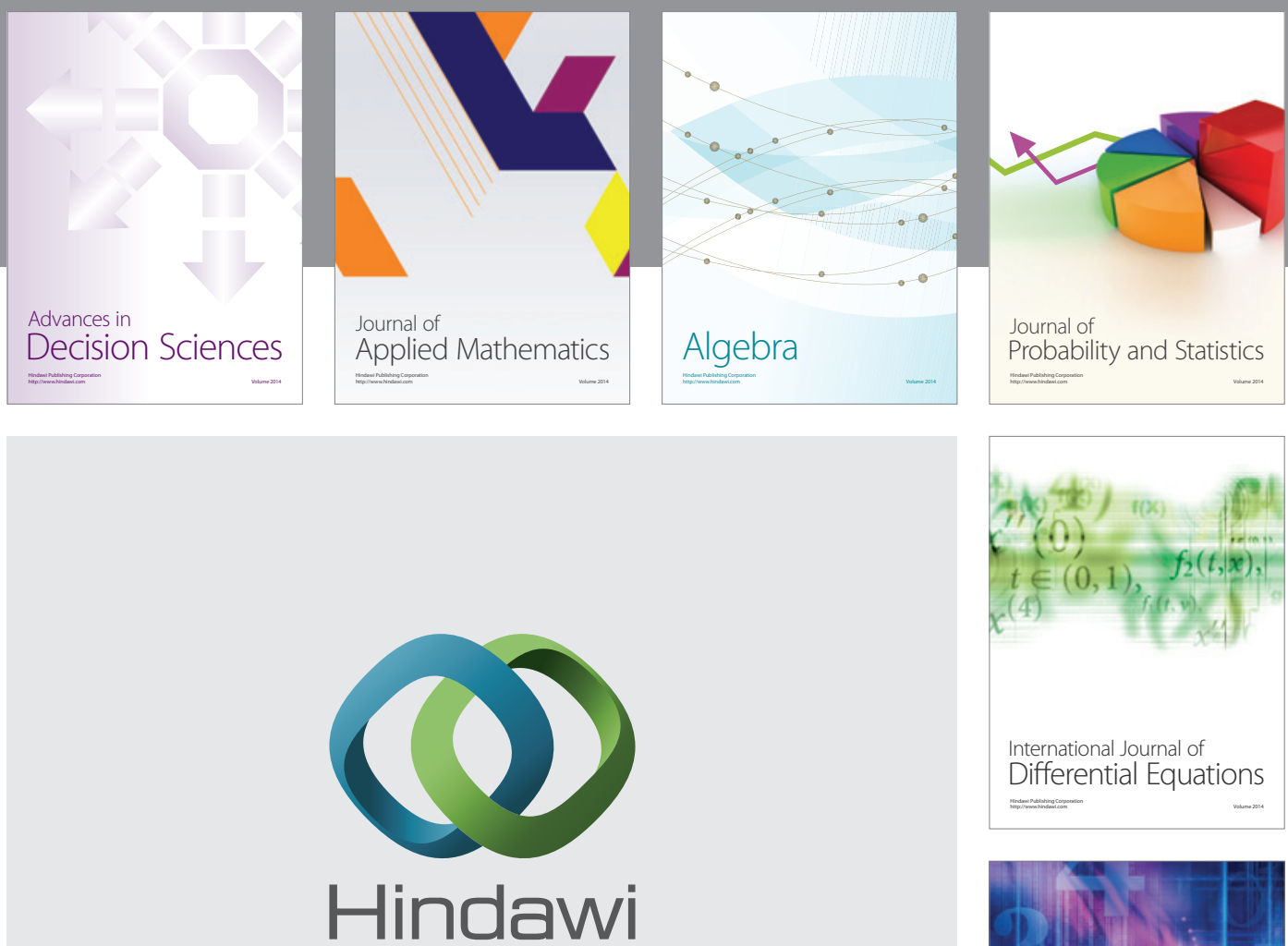

Submit your manuscripts at http://www.hindawi.com
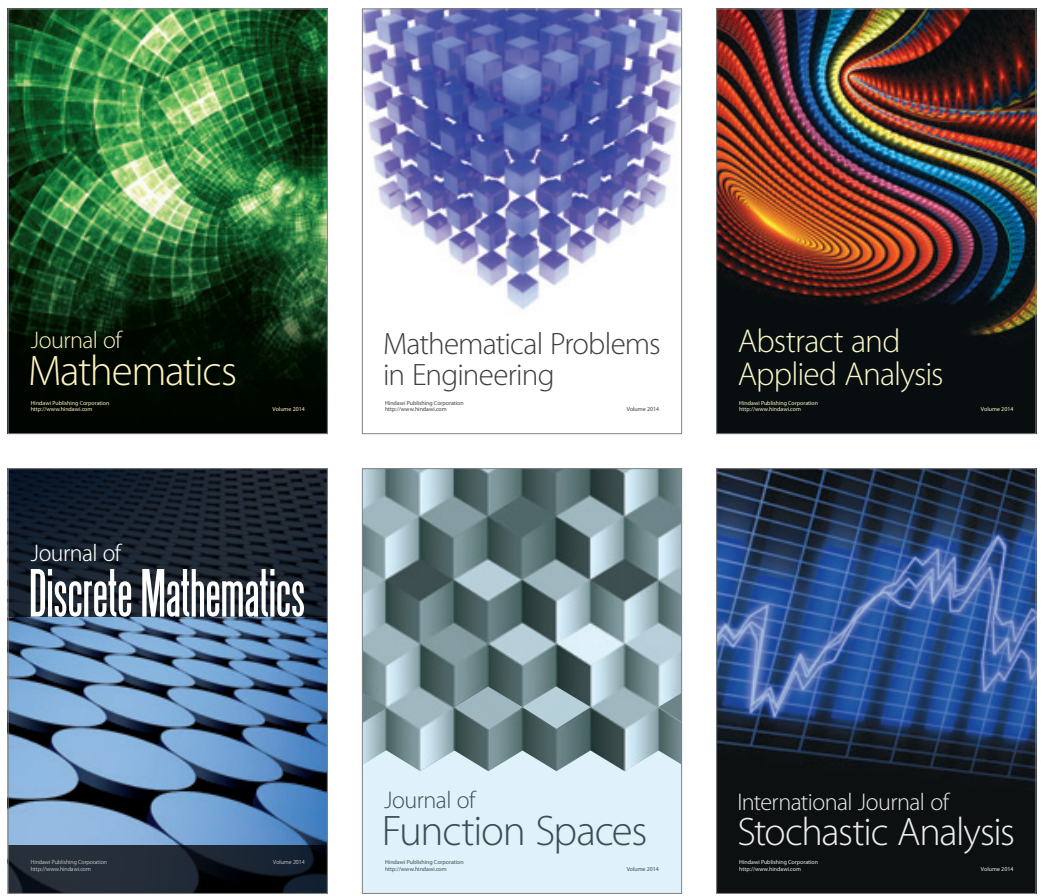

Journal of

Function Spaces

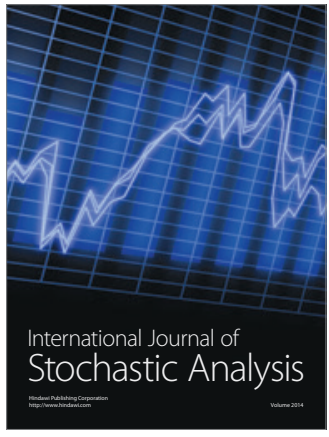


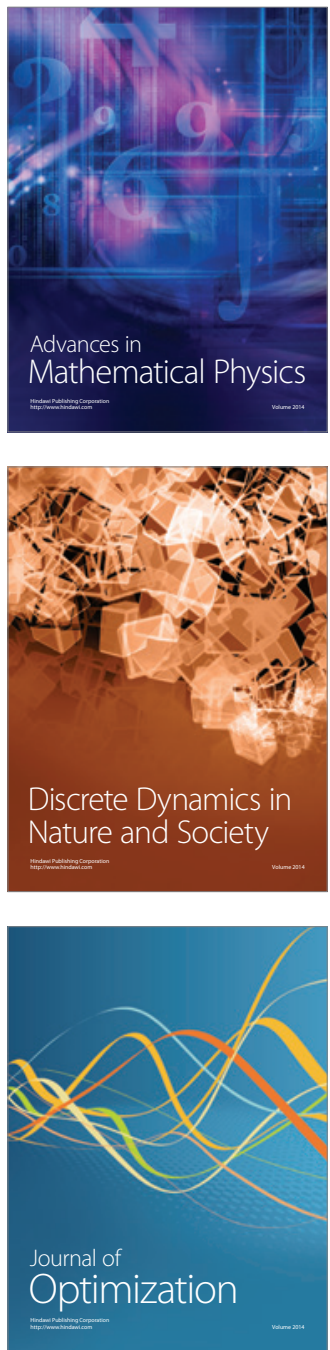